\title{
Subnormal closure of a homomorphism
}

\author{
Emmanuel D. Farjoun • Yoav Segev
}

Received: 7 May 2014 / Accepted: 19 November 2014 / Published online: 5 December 2014

(C) Tbilisi Centre for Mathematical Sciences 2014

\begin{abstract}
Let $\varphi: \Gamma \rightarrow G$ be a homomorphism of groups. In this paper we introduce the notion of a subnormal map (the inclusion of a subnormal subgroup into a group being a basic prototype). We then consider factorizations $\Gamma \stackrel{\psi}{\rightarrow} M \stackrel{n}{\rightarrow} G$ of $\varphi$, with $n$ a subnormal map, namely a finite composition of the underlying maps of crossed modules. We search for a universal such factorization. When $\Gamma$ and $G$ are finite we show that such universal factorization exists: $\Gamma \rightarrow \Gamma_{\infty} \rightarrow G$, where $\Gamma_{\infty}$ is a hypercentral extension of the subnormal closure $\mathcal{C}$ of $\varphi(\Gamma)$ in $G$ (i.e. the kernel of the extension $\Gamma_{\infty} \rightarrow \mathcal{C}$ is contained in the hypercenter of $\left.\Gamma_{\infty}\right)$. This is closely related to the a relative version of the Bousfield-Kan $\mathbb{Z}$-completion tower of a space. The group $\Gamma_{\infty}$ is the inverse limit of the normal closures tower of $\varphi$ introduced by us in a recent paper. We prove several stability and finiteness properties of the tower and its inverse limit $\Gamma_{\infty}$
\end{abstract}

Keywords Subnormal map - Normal closures tower · Hypercentral group extension Mathematics Subject Classification Primary 20E22; Secondary 20J06 - 20F28 • 18A40

Communicated by Vladimir Vershinin.

E. D. Farjoun $(\varangle)$

Department of Mathematics, Hebrew University of Jerusalem,

Givat Ram, Jerusalem 91904, Israel

e-mail: farjoun@math.huji.ac.il

Y. Segev

Department of Mathematics, Ben Gurion University,

Beer Sheva 84105, Israel

e-mail: yoavs@math.bgu.ac.il 


\section{Introduction}

Throughout this note $\varphi: \Gamma \rightarrow G$ is a homomorphism of groups. In a previous paper [6] we considered the notion of the free crossed module closure of $\varphi$ (which in [6] we called the free normal closure $\Gamma^{\varphi}$ of $\varphi$, and is related to [3,4]). We also discussed in [6] the free crossed module closures tower $\left\{\Gamma_{i, \varphi}\right\}_{i=1}^{\infty}$ of $\varphi$ (called the free normal closures tower in [6], see Eq. (1.1) below). In this paper we study the behavior and the properties of the inverse limit $\Gamma_{\infty, \varphi}$ of the free crossed module closures tower of $\varphi$. This tower generalizes and connects the quotients of the lower central series $\Gamma / \gamma_{i}(\Gamma), i=1,2,3, \ldots$, gotten here for $G=1$, and the descending series of successive normal closures of a subgroup $H$ of $G$ (see Sect. 2). Notice that in the case $G=1$, the group $\Gamma_{\infty, \varphi}$ is the nilpotent completion of $\Gamma$. Thus for an arbitrary map $\varphi$, the group $\Gamma_{\infty, \varphi}$ can be thought of as a relative nilpotent completion associated with a homomorphism rather than with a group. Some of the results here support this point of view.

Let us recall from $[6$, Sect. 4$]$ that the crossed module closures tower (we often omit the word "free") associated with a homomorphism $\varphi$ is a tower of groups as follows:

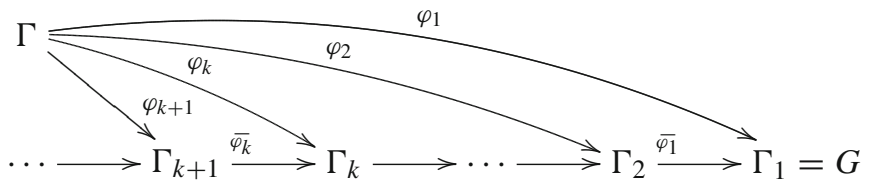

where $\overline{\varphi_{i}}$ is a normal map (throughout this paper, as well as in [6], this is our terminology for the underling map of a crossed module structure), and where $\Gamma_{i+1}=\Gamma_{i+1, \varphi}$ is the (free) crossed module closure $\Gamma_{i}^{\varphi_{i}}$ of the map $\Gamma \stackrel{\varphi_{i}}{\rightarrow} \Gamma_{i}$, for all $i \geq 1$. The group $\Gamma_{\infty}=\Gamma_{\infty, \varphi}$, the stability of the tower $\left\{\Gamma_{i, \varphi}\right\}$ and its relation to the groups $\Gamma, G$ and the map $\varphi$, are thus the main topics of the present study.

One way to think about the above tower is that it represents an attempt to factor the map $\varphi$, in a universal way, into a composition of "simpler maps" (i.e. normal maps). This, of course, cannot be done in general, and $\Gamma_{\infty, \varphi}$ is a kind of "hybrid" of $\Gamma$ and $G$ giving a factorization $\Gamma \rightarrow \Gamma_{\infty, \varphi} \rightarrow G$. Passing to topological spaces via the classifying space construction we get a map $B \Gamma \rightarrow B G$. The present result shows that for finite groups $\Gamma, G$ this map has a finite "relative" Bousfield-Kan tower of principal fibrations [2] whose fibres are, in general, neither connected nor nilpotent groups:

$$
B \Gamma_{\infty}=B \Gamma_{n} \rightarrow B \Gamma_{n-1} \rightarrow \cdots \rightarrow B \Gamma_{2} \rightarrow B G
$$

with a terminal term $B \Gamma_{\infty}=B \Gamma_{n} \rightarrow B G$ being the universal space, under $B \Gamma$ for which there is such a tower of principal fibrations. This raises the question of finding such universal decompositions of more general maps of spaces $X \rightarrow Y$. Notice that we have an induced map $\varphi_{\infty}: \Gamma \rightarrow \Gamma_{\infty}$; we often ask how far is this map from an isomorphism.

One quick corollary of our main results concerns a map of nilpotent groups: 
Theorem 1 Let $\varphi: \Gamma \rightarrow G$ be a homomorphism of nilpotent groups, then $\varphi_{\infty}: \Gamma \rightarrow$ $\Gamma_{\infty}$ is an isomorphism.

Theorem 1 is Corollary 2.13(2). This corollary can be viewed as a version for any map of nilpotent groups, of the well known property that any subgroup of a nilpotent group is subnormal, i.e. equal to its subnormal closure.

In this spirit we have for any homomorphism of finite groups:

Theorem 2 Let $\varphi: \Gamma \rightarrow G$ be a homomorphism of finite groups. Then $\varphi_{\infty}$ induces an isomorphism of the descending central series quotients, $\Gamma / \gamma_{i}(\Gamma) \cong \Gamma_{\infty} / \gamma_{i}\left(\Gamma_{\infty}\right)$, for all $i \geq 1$.

Theorem 2 is proved in Proposition 2.14. We note that by previous results, this is certainly not true for a general map of groups, e.g., when $\Gamma$ is a free group with infinitely many generators and $G=1$.

Our next result extends a partial result in [6] to the general finite case. An estimate of the size of $\Gamma_{\infty}$ is given below in Theorem 2.11 .

Theorem 3 If $\varphi: \Gamma \rightarrow G$ is a homomorphism of finite groups, then $\Gamma_{\infty, \varphi}$ is a finite group.

We note that even when $\varphi$ is the trivial map between two finite cyclic groups, the groups $\Gamma_{i, \varphi}$ grow indefinitely in size with $i$, but their inverse limit is finite, and is isomorphic to the domain in this case (Theorem 1).

Recall that for a finite group $G$ and a subgroup $H \leq G$, the subnormal closure of $H$ in $G$ is the smallest subnormal subgroup of $G$ containing $H$. Thus Theorem 3 is again an extension to any map of finite groups, of the trivial observation that the subnormal closure of a subgroup of a finite group is well defined and finite. This result complements the dual result proved in [6] for the tower of injective normalizers of a map of finite groups.

The next theorem characterizes, for maps of finite groups, the factorization $\Gamma \rightarrow$ $\Gamma_{\infty} \rightarrow G$ as a universal one among all subnormal factorizations (see below), the proof is given in Sect. 3 .

The following is a basic definition:

Definition 4 A subnormal map $n: M \rightarrow G$, is a homomorphism such that there exists a finite series of normal maps $n_{i}: M_{i+1} \rightarrow M_{i}, 1 \leq i \leq k$, with $M_{1}=G$, whose composition is $n$.

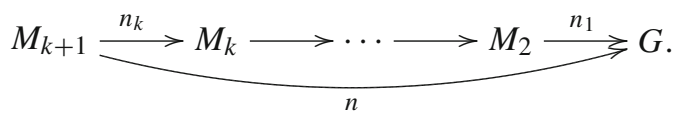

Notice that the image subgroup $n\left(M_{k+1}\right)$ is subnormal in $G$, and the precise crossed module structure of the maps $n_{i}$ is immaterial.

For example, any map of nilpotent groups is a subnormal map. Note that in Theorem 5 and its Corollary 6 below the crossed module structure of the normal maps is absent. 
Theorem 5 If $\varphi: \Gamma \rightarrow G$ be a homomorphism of finite groups then the factorization $\Gamma \stackrel{\varphi_{\infty}}{\longrightarrow} \Gamma_{\infty, \varphi} \rightarrow G$ is the universal initial factorization of $\varphi$, among all factorizations $\Gamma \rightarrow S \rightarrow G$ of $\varphi$, with the right map $S \rightarrow G$ being a subnormal map; namely, it maps uniquely to every such factorization via $\Gamma_{\infty, \varphi} \rightarrow S$.

An immediate consequence of Theorem 5 is the following:

Corollary 6 Suppose $\varphi: \Gamma \rightarrow G$ is a subnormal map, then $\Gamma_{\infty, \varphi} \cong \Gamma$.

Proof This follows directly from the uniqueness in Theorem 5. Indeed, replacing $S$ with $\Gamma$, the map $\rho: \Gamma_{\infty, \varphi} \rightarrow \Gamma$ has to be the inverse of the map $\varphi$ as their composition $\rho \circ \varphi$ (maps acting on the right) is the unique map of Theorem 5 with $S$ replaced by $\Gamma_{\infty, \varphi}$, and this map (being unique) has to be the identity.

Main tools Here we sum-up the main technical tools for proving the above results. They consist of showing that the limiting group $\Gamma_{\infty, \varphi}$ does not change up to a canonical isomorphism, when one changes the domain or range of $\varphi$ in certain controlled ways.

First we consider changing the range via factoring our map $\varphi$ through any subnormal map $M \rightarrow G$.

The following theorem is one of our main tools showing we can perform the above mentioned replacement:

Theorem 7 Let $\varphi: \Gamma \rightarrow G$ be a homomorphism and let $\Gamma \stackrel{\psi}{\rightarrow} M \stackrel{n}{\rightarrow}$ G be a factorization of $\varphi$ such that $n: M \rightarrow G$ is a subnormal map. Then $\Gamma_{\infty, \varphi} \cong \Gamma_{\infty, \psi}$.

Theorem 7 is proved right after Proposition 2.4.

The next result shows that one can factor out a certain portion $\mathcal{K}$ of the kernel $K$ of $\varphi$ and still obtain that $\Gamma_{\infty, \varphi} \cong(\Gamma / \mathcal{K})_{\infty, \rho}$, where $\rho: \Gamma / \mathcal{K} \rightarrow G$ is the map induced by $\varphi$.

To define $\mathcal{K}$, define the descending series of successive commutators of $K$ with $\Gamma$ by $K_{1}=K, K_{2}=[\Gamma, K]$, and in general $K_{i+1}=\left[\Gamma, K_{i}\right]$. If this series terminates after a finite number of steps, we let $\mathcal{K}$ be the final member of this series.

Proposition 8 Let $\varphi: \Gamma \rightarrow G$ be a homomorphism, and suppose that the descending series of successive commutators of $K=\operatorname{ker} \varphi$ with $\Gamma$ terminates after a finite number of steps. Let $\mathcal{K}$ be the terminal member of this series. Then $\Gamma_{\infty, \varphi} \cong(\Gamma / \mathcal{K})_{\infty, \rho}$, where $\rho: \Gamma / \mathcal{K} \rightarrow G$ is the map induced by $\varphi$.

Proposition 8 is Proposition 2.9(2). Notice that in the notation of Proposition 8, the kernel of $\rho$ is $K / \mathcal{K}$ and hence $\operatorname{ker} \rho$ is contained in some member of the ascending central series of $\Gamma / \mathcal{K}$. In Example 4.1 we show however that the nilpotency class of $K / \mathcal{K}$ cannot be bounded.

\section{Equivalence of closures towers}

In this section we prove the results of the introduction, and analyze further the group $\Gamma_{\infty, \varphi}$. Our main tool is to compare the crossed module closures tower $\left\{\Gamma_{i, \varphi}\right\}$ to towers $\left\{\Lambda_{i, \rho}\right\}$, for various homomorphism $\rho: \Lambda \rightarrow H$, which are, of course, related to $\varphi$. 
As noted above, throughout this paper $\varphi: \Gamma \rightarrow G$ is a fixed homomorphism of groups. We use the following notation. $K=\operatorname{ker} \varphi$ and $\Gamma^{\varphi}$ is the (free) crossed module closure of $\varphi$. This, we recall, is the universal crossed module factorization $\Gamma \stackrel{c_{\varphi}}{\longrightarrow}$ $\Gamma^{\varphi} \stackrel{\bar{\varphi}}{\rightarrow} G$ of $\varphi$ with the right map equipped with a universal (free) crossed module structure.

As in diagram (1.1), $\left\{\Gamma_{i}\right\}=\left\{\Gamma_{i, \varphi}\right\}$ is the crossed module closures tower of $\varphi$, and $\varphi_{i}, \overline{\varphi_{i}}$ are as in diagram (1.1). $\Gamma_{\infty}=\lim \Gamma_{i}$ and $\varphi_{\infty}: \Gamma \rightarrow \Gamma_{\infty}$ is the natural map.

Terminology 2.1 Let $k \geq 0$ be an integer, and let $\left\{H_{i}\right\}_{i=k}^{\infty}$ be a decreasing or increasing series of groups. We say that $\left\{H_{i}\right\}$ terminates if there exists an integer $t \geq k$, such that $H_{t}=H_{t+1}=H_{t+2}=\cdots$. In this case we call $H_{t}$ the terminal member of the series and say that the series terminates at $H_{t}$.

Recall from [9, p. 385] the notion of the series of successive normal closures-in the usual sense-of a subgroup $H \leq G$ in $G$. This is the descending series defined by $C_{0}=G, C_{1}=\left\langle H^{C_{0}}\right\rangle$, and in general $C_{i+1}=\left\langle H^{C_{i}}\right\rangle$.

Notation 2.2 (1) The series of successive normal closures of $\varphi(\Gamma)$ in $G$ will be denoted $G=C_{0}, C_{1}, C_{2} \ldots$ If this series terminates, then we denote by $\mathcal{C}$ its terminal member.

(2) Let $K:=\operatorname{ker} \varphi$ and define the descending series of successive commutators of $K$ with $\Gamma$ by $K_{1}=K, K_{2}=[\Gamma, K]$, and in general $K_{i+1}=\left[\Gamma, K_{i}\right]$. If this series terminates, then we denote by $\mathcal{K}$ its terminal member.

Next $H=\gamma_{1}(H), \gamma_{2}(H), \ldots$ denotes the descending central series of the group $H$. If this series terminates, then $\gamma_{\infty}(H)$ denotes the terminal member of this series. Finally, the ascending central series of $H$ is denoted $1=Z_{0}(H), Z_{1}(H), \ldots$, and the same convention as above applies for the notation $Z_{\infty}(H)$.

We refer the reader to [6, Sect. 2] for the notions of a normal map and of a crossed module morphism (which in [6] is called a normal morphism), where references to previous work on this subject in given. In [6, Sect. 3] the reader will find some basic properties of $\Gamma^{\varphi}$ and in [6, Sect. 4] some basic properties of the crossed module closures tower of $\varphi$.

Let us start by recalling the naturality of the crossed module closures tower.

Lemma 2.3 Any commutative diagram

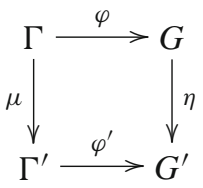

induces a commutative diagram between the crossed module closures tower of $\varphi$ and $\varphi^{\prime}$ : 
Namely if we set $\Gamma_{1}:=G, \Gamma_{1}^{\prime}=G^{\prime}$ and $\eta_{1}:=\eta$, we have the following commutative diagram:

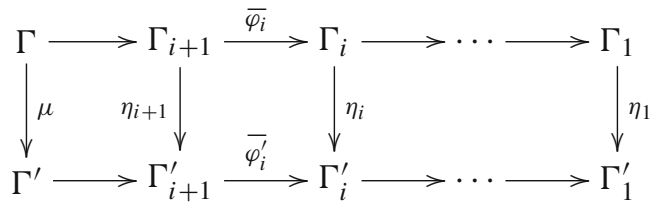

Thus there is a canonical map $\eta_{\infty}: \Gamma_{\infty} \rightarrow \Gamma_{\infty}^{\prime}$ and equalities: $\varphi_{i} \circ \eta_{i}=\mu \circ \varphi_{i}^{\prime}$, for all $i \geq 1$.

Proof Take in diagram (2.2) of [6], $M^{\prime}=\Gamma_{2}^{\prime}, \psi^{\prime}=\varphi_{2}^{\prime}$ and $n^{\prime}=\overline{\varphi_{1}^{\prime}}$, and use the universality properties of $\Gamma_{2}$ to obtain $\eta_{2}$. Then proceed in this manner replacing each time $\Gamma_{i}, \Gamma_{i}^{\prime}$, with $\Gamma_{i+1}, \Gamma_{i+1}^{\prime}$, respectively.

The following proposition will be used in the proof of Theorem 7 of the introduction. It addresses the replacement of $G$ by the domain of any subnormal map $M \rightarrow G$ that factors our map $\varphi$. In that case the map of towers in diagram (2.3) is in fact a proisomorphism of towers:

Proposition 2.4 Let $\Gamma \stackrel{\psi}{\rightarrow} M \stackrel{n}{\rightarrow} G$ be a factorization of $\varphi$ with $n$ a normal map. Then the above commutative diagram (2.2) extends to a natural commutative diagram in which the upper (resp. lower) raw is the crossed module closures tower of $\Gamma \stackrel{\psi}{\rightarrow} M$ $($ resp. $\Gamma \stackrel{\varphi}{\rightarrow} G)$ :

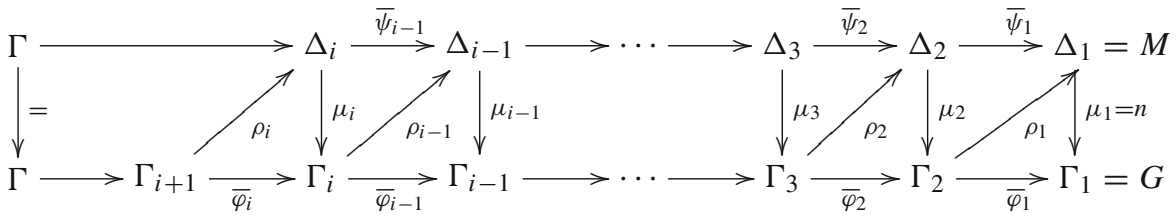

Proof We need the following lemma which is an obvious analog of the similar situation for two normal subgroup of the same group:

Lemma 2.5 If the following is a commutative diagram

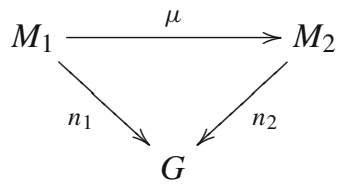

where $n_{i}$ are equipped with a crossed module structure, for $i=1,2$, and $\mu$ is a crossed module morphism, then there exists a crossed module structure on $\mu$. 
Proof Here and throughout this paper, the crossed module structure is denoted exponentially. Define an action of $M_{2}$ on $M_{1}$ by $m_{1}^{m_{2}}=m_{1}^{n_{2}\left(m_{2}\right)}, m_{i} \in M_{i}$. Then it is immediate that $\mu$ becomes a normal map with the above crossed module structure.

As usual let $\Gamma_{1}:=G$ and $\varphi_{1}:=\varphi$. Consider the crossed module closures tower corresponding to $\psi$. So the groups $\Gamma_{i}$ in diagram (1.1) are replace by $\Delta_{i}$ and the maps $\varphi_{i}, \bar{\varphi}_{i}$ are replaced by $\psi_{i}, \bar{\psi}_{i}$, respectively. Thus $\Delta_{1}=M$ and $\psi_{1}=\psi$. Set $\mu_{1}=n$, thus $\mu_{1}: \Delta_{1} \rightarrow \Gamma_{1}$ is a normal map.

We show that there are normal maps $\mu_{i}, \rho_{i}, i \geq 1$, as in diagram (2.3) such that

$$
\varphi_{i+1} \circ \rho_{i}=\psi_{i} \text { and } \psi_{i} \circ \mu_{i}=\varphi_{i}, \quad \text { for all } i \geq 1 \text {. }
$$

Now since $\Gamma \stackrel{\psi_{1}}{\longrightarrow} \Delta_{1} \stackrel{\mu_{1}}{\longrightarrow} \Gamma_{1}$ is a factorization of $\varphi_{1}$, by the universality property of $\Gamma_{2}$, there exists a crossed module morphism $\rho_{1}: \Gamma_{2} \rightarrow \Delta_{1}$ for the lower right triangle, and such that $\varphi_{2} \circ \rho_{1}=\psi_{1}$. By Lemma 2.5, $\rho_{1}$ is a normal map.

Let now $i \geq 2$, and suppose that $\rho_{i-1}$ and $\mu_{i-1}$ were defined, they are normal maps, diagram (2.3) is commutative up to the $(i-1)$-step, and Eq. (2.4) holds. By Eq. (2.4) we have $\varphi_{i} \circ \rho_{i-1}=\psi_{i-1}$. By the universality of $\Delta_{i}$, and since $\rho_{i-1}$ is a normal map, there exists a map $\mu_{i}: \Delta_{i} \rightarrow \Gamma_{i}$ such that

(a) $\psi_{i} \circ \mu_{i}=\varphi_{i}$.

(b) $\mu_{i} \circ \rho_{i-1}=\bar{\varphi}_{i}$, and $\mu_{i}$ is a crossed module morphism for the right triangle.

By Lemma 2.5, $\mu_{i}$ is a normal map.

Next, by the universality property of $\Gamma_{i+1}$, by (a), and since $\mu_{i}$ is a normal map, there exists a crossed module morphism $\rho_{i}: \Gamma_{i+1} \rightarrow \Delta_{i}$ for the lower right triangle, this triangle is commutative $\left(\rho_{i} \circ \mu_{i}=\overline{\varphi_{i}}\right)$ and $\varphi_{i+1} \circ \rho_{i}=\psi_{i}$. Again by Lemma 2.5, $\rho_{i}$ is a normal map. This completes the induction step and the proof of the proposition.

Proof of Theorem 7 Consider the subnormal series of normal maps

$$
M_{k+1} \stackrel{n_{k}}{\longrightarrow} M_{k} \longrightarrow \cdots \longrightarrow M_{2} \stackrel{n_{1}}{\longrightarrow} G=M_{1}
$$

where $k \geq 1, M_{k+1}=M, M_{1}=G$, and $n_{t}$ is a normal map, for all $1 \leq t \leq k$. Let $\psi_{t}: \Gamma \rightarrow M_{t}$ be the maps defined by $\psi_{k+1}=\psi$, and for $1 \leq t \leq k$, let $\psi_{t}=\psi \circ n_{k} \circ \cdots \circ n_{t}\left(\right.$ so $\left.\psi_{1}=\varphi\right)$.

The universality property of the inverse limit and Proposition 2.4 imply that $\Gamma_{\infty, \psi_{t}} \cong \Gamma_{\infty, \psi_{t+1}}$, for all $1 \leq t \leq k$, so since $\Gamma_{\infty, \varphi}=\Gamma_{\infty, \psi_{1}}$ and $\Gamma_{\infty, \psi}=\Gamma_{\infty, \psi_{k+1}}$, the theorem holds.

As a corollary to Theorem 7 we get

Proposition 2.6 (1) Let $H$ be a subnormal subgroup of $G$ containing $\varphi(\Gamma)$. Then $\Gamma_{\infty, \varphi} \cong \Gamma_{\infty, \psi}$, where $\psi: \Gamma \rightarrow H$ is the restriction of $\varphi$ in the range; 
(2) if the series $\left\{C_{i}\right\}$ terminates, then $\Gamma_{\infty, \varphi}=\Gamma_{\infty, \psi}$, where $\psi: \Gamma \rightarrow \mathcal{C}$ is the restriction of $\varphi$ in the range.

Proof Let $n: H \hookrightarrow G$ be the inclusion map. Then $n$ is a subnormal map, and $\Gamma \stackrel{\psi}{\rightarrow}$ $H \stackrel{n}{\rightarrow} G$ is a factorization of $\varphi$. Hence (1) follows from Theorem 7, and (2) is immediate from (1).

We now turn our attention to the kernel $K$.

Lemma 2.7 Let $L \leq \operatorname{ker} c_{\varphi}$ be a normal subgroup of $\Gamma$. Let $\rho: \Gamma / L \rightarrow G$ be the homomorphism induced by $\varphi$, and let $\beta: \Gamma / L \rightarrow \Gamma^{\varphi}$ be the homomorphism induced by $c_{\varphi}$. Then $\Gamma^{\varphi}$ and $(\Gamma / L)^{\rho}$ are naturally isomorphic.

Proof Consider the following commutative diagram. We show that $u$ is an isomorphism whose inverse is $v$.

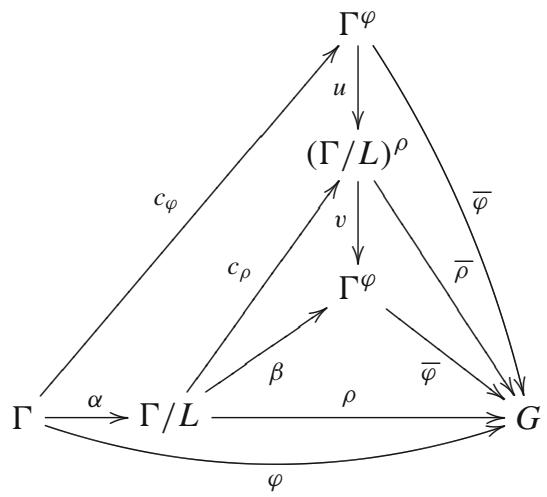

here $\alpha \circ \rho=\varphi, \alpha \circ \beta=c_{\varphi}$, and $u$ and $v$ are the unique crossed module morphisms obtained from the universality properties of $\Gamma^{\varphi}$ and $(\Gamma / L)^{\rho}$, respectively.

Notice that $\alpha \circ \beta \circ \bar{\varphi}=c_{\varphi} \circ \bar{\varphi}=\varphi=\alpha \circ \rho$. Since $\alpha$ is surjective we see that $\beta \circ \bar{\varphi}=\rho$. By the universality property of $(\Gamma / L)^{\rho}$ we get the map $v$. Also, $\left(\alpha \circ c_{\rho}\right) \circ \bar{\rho}=\alpha \circ \rho=\varphi$. By the universality property of $\Gamma^{\varphi}$ we get the map $u$.

Next we have $c_{\varphi} \circ u \circ v=\alpha \circ c_{\rho} \circ v=\alpha \circ \beta=c_{\varphi}$. Also $u \circ v \circ \bar{\varphi}=u \circ \bar{\rho}=\bar{\varphi}$. Hence $u \circ v=1_{\Gamma^{\varphi}}$, by the uniqueness in the universality property of $\Gamma^{\varphi}$.

Further, $\alpha \circ c_{\rho} \circ v \circ u=\alpha \circ \beta \circ u=c_{\varphi} \circ u=\alpha \circ c_{\rho}$. Since $\alpha$ is surjective, $c_{\rho} \circ v \circ u=c_{\rho}$. Also $v \circ u \circ \bar{\rho}=v \circ \bar{\varphi}=\bar{\rho}$. So, as above, $v \circ u$ is the identity map of $(\Gamma / L)^{\rho}$.

As a corollary we get

Corollary 2.8 Let $L \leq \operatorname{ker} c_{\varphi}$ be a normal subgroup of $\Gamma$, and let $\rho: \Gamma / L \rightarrow G$ be the homomorphism induced by $\varphi$. Then $\Gamma / L \stackrel{\beta}{\rightarrow} \Gamma^{\varphi} \stackrel{\bar{\varphi}}{\rightarrow} G$, where $\beta$ and $\bar{\varphi}$ are as in diagram (2.5), is the universal crossed module factorization of $\rho$.

Proof This follows from Lemma 2.7, because in diagram (2.5) we may assume that $c_{\rho}=\beta,(\Gamma / L)^{\rho}=\Gamma^{\varphi}$ and $\bar{\rho}=\bar{\varphi}$. 
In particular we get Proposition 8 of the introduction as part (2) of the following:

Proposition 2.9 Let $L \leq \Gamma$ be a subgroup with $L \leq \operatorname{ker} \varphi_{i}$, for all integers $i \geq 1$. Let $\rho: \Gamma / L \rightarrow G$ be the map induced by $\varphi$, let $\left\{(\Gamma / L)_{i}\right\}=\left\{(\Gamma / L)_{i, \rho}\right\}$ be the crossed module closures tower of $\rho$, and let $\rho_{i}, \overline{\rho_{i}}$ be the maps for the tower $\left\{(\Gamma / L)_{i}\right\}$. Then

(1) there is a natural isomorphism $\Gamma_{i} \cong(\Gamma / L)_{i}$, where $\rho_{i}: \Gamma / L \rightarrow(\Gamma / L)_{i}$ is the map induced by $\varphi_{i}$, and $\overline{\varphi_{i}}=\overline{\rho_{i}}$, for all $i \geq 1$. In particular $\Gamma_{\infty} \cong(\Gamma / L)_{\infty}$;

(2) if the series $\left\{K_{i}\right\}$ terminates at $\mathcal{K}$, then (1) above holds for $L:=\mathcal{K}$.

Proof Part (1) is immediate from Corollary 2.8. For (2) note that $\varphi_{i+1}\left(\operatorname{ker} \varphi_{i}\right) \leq$ $\operatorname{ker} \overline{\varphi_{i}} \leq Z\left(\Gamma_{i+1}\right)$. It follows by induction on $i$, that $K_{i} \leq \operatorname{ker} \varphi_{i}$, for all $i \geq 1$. This implies that if the series $\left\{K_{i}\right\}$ terminates, then $\mathcal{K} \leq \operatorname{ker} \varphi_{i}$, for all $i \geq 1$, so (2) follows from (1).

Combining Propositions 2.9(2) and 2.6(2) we get:

Corollary 2.10 Assume that both the series $\left\{C_{i}\right\}$ and the series $\left\{K_{i}\right\}$ terminate at $\mathcal{C}$ and $\mathcal{K}$, respectively. Then there is an isomorphism $\Gamma_{\infty, \varphi} \cong(\Gamma / \mathcal{K})_{\infty, \psi}$, where $\psi: \Gamma / \mathcal{K} \rightarrow \mathcal{C}$, is the map induced by $\varphi$.

Proof By Proposition 2.9(2), $\Gamma_{\infty, \varphi}=(\Gamma / \mathcal{K})_{\infty, \rho}$, where $\rho: \Gamma / \mathcal{K} \rightarrow G$ is the map induced by $\varphi$. Then, by Proposition 2.6(2) (with $\Gamma / \mathcal{K}$ in place of $\Gamma$ ), $(\Gamma / \mathcal{K})_{\infty, \rho}=$ $(\Gamma / \mathcal{K})_{\infty, \psi}$.

The following theorem proves in particular the assertion of Theorem 3 of the introduction.

Theorem 2.11 Let $\varphi: \Gamma \rightarrow G$ be a homomorphism of finite groups. Let $\rho: \Gamma \rightarrow \mathcal{C}$ and let $\psi: \Gamma / \mathcal{K} \rightarrow \mathcal{C}$ be the maps induced by $\varphi$. Then

(1) $\Gamma_{\infty, \varphi} \cong \Gamma_{\infty, \rho}=(\Gamma / \mathcal{K})_{\infty, \psi}$;

(2) the crossed module closures series $\left\{\Gamma_{i, \rho}\right\}$ and the series $\left\{(\Gamma / \mathcal{K})_{i, \psi}\right\}$ terminate, hence $\Gamma_{\infty, \varphi} \cong \Gamma_{t_{1}, \rho} \cong(\Gamma / \mathcal{K})_{t_{2}, \psi}$, where $\Gamma_{t_{1}, \rho}$ and $(\Gamma / \mathcal{K})_{t_{2}, \psi}$ are the terminal members of the respective series;

(3) $\Gamma_{\infty, \varphi}$ is finite and $\left|\Gamma_{\infty, \varphi}\right| \leq|\Gamma / \mathcal{K}| \cdot g(|\mathcal{C}|)$, where $g(1)=1$, and for any integer $t \geq 2$,

$$
g(t)=t^{k} \text {, where } k=\frac{1}{2}\left(\log _{p} t+1\right) \text { and } p \text { is the least prime divisor of } t .
$$

Proof Part (1) follows from Proposition 2.6(2) and Corollary 2.10. Then, by definition, $\mathcal{C}=\left\langle\varphi(\Gamma)^{\mathcal{C}}\right\rangle$, so parts (2) and (3) follow from [6, Theorem 4.1].

Furthermore we can use Corollary 2.10 to prove the following lemma, which leads to the proof of Theorem 1 of the introduction.

Lemma 2.12 Assume that $\varphi(\Gamma)$ is subnormal in $G$ and that the series $\left\{K_{i}\right\}$ (see Notation 2.2(2)) terminates. Then $\Gamma_{\infty}=\Gamma / \mathcal{K}$. 
Proof Since $\varphi(\Gamma)$ is subnormal in $G$, we have $\mathcal{C}=\varphi(\Gamma)$. By Proposition 2.6(2), to evaluate $\Gamma_{\infty}$, we may assume that $\varphi$ is surjective. By [6, Corollary 3.9(2)], $\Gamma_{2}=$ $\Gamma /[\Gamma, K]$, and $\varphi_{2}: \Gamma \rightarrow \Gamma_{2}$ is the canonical map. Iterating on [6, Corollary 3.9(2)] we see that $\Gamma_{i}=\Gamma / K_{i}$, for all $i \geq 2$, thus $\Gamma_{\infty}=\Gamma / \mathcal{K}$.

Part (2) of the following corollary is Theorem 1 of the introduction.

Corollary 2.13 (1) If $\varphi(\Gamma)$ is subnormal in $G$ and $K$ is contained in $Z_{i}(\Gamma)$ for some integer $i \geq 0$ (which holds if $\varphi$ is injective), then $\Gamma_{\infty}=\Gamma$;

(2) if $\Gamma$ and $G$ are nilpotent, then $\Gamma_{\infty}=\Gamma$.

Proof Part (1) is an immediate consequence of Lemma 2.12, since under the hypotheses of (1), $\mathcal{K}=1$. Then (2) follows from (1).

We now turn to the nilpotent quotients of $\Gamma_{\infty}=\Gamma_{\infty, \varphi}$, and prove Theorem 2 of the introduction.

Proposition 2.14 Let $k \geq 1$. The $\operatorname{map} \varphi_{(\infty, k)}: \Gamma / \gamma_{k}(\Gamma) \rightarrow \Gamma_{\infty} / \gamma_{k}\left(\Gamma_{\infty}\right)$ induced by the canonical map $\varphi_{\infty}: \Gamma \rightarrow \Gamma_{\infty}$ is injective. If $\Gamma$ and $G$ are finite, then it induces an isomorphism $\Gamma / \gamma_{\infty}(\Gamma) \cong \Gamma_{\infty} / \gamma_{\infty}\left(\Gamma_{\infty}\right)$.

Proof Let $\psi: \Gamma / \gamma_{k}(\Gamma) \rightarrow G / \gamma_{k}(G)$ be the map induced by $\varphi$. By naturality (Lemma 2.3) we have the following commutative diagram

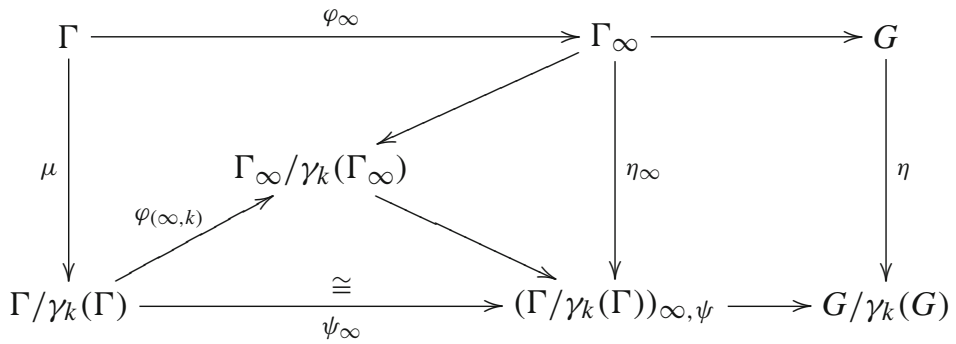

where the map $\eta_{\infty}$ is obtained from Lemma 2.3 , and where $\psi_{\infty}$ is an isomorphism by Corollary 2.13(2). The diagram is commutative since $\mu$ is surjective. Since $\psi_{\infty}$ is an isomorphism, $\varphi_{(\infty, k)}$ is injective.

Assume that $\Gamma$ and $G$ are finite. By Proposition 2.6 (2) we may assume that $G=\mathcal{C}$, so $G=\left\langle\varphi(\Gamma)^{G}\right\rangle$. Also, by Theorem 2.11(2), the series $\left\{\Gamma_{i}\right\}$ terminates, so $\Gamma_{\infty}=\Gamma_{t}$, for some $t \geq 1$, and now $\varphi_{\infty}=\varphi_{t}$. By [6, Lemma 4.2(1)], $\Gamma_{\infty}=\Gamma_{t}=\left\langle\varphi_{t}(\Gamma)^{\Gamma_{t}}\right\rangle$. Hence the conjugates of the image of $\varphi_{t}(\Gamma)$ in $\Gamma_{t} / \gamma_{k}\left(\Gamma_{t}\right)$ generates it. By [6, Lemma 4.4], the image of $\varphi_{t}(\Gamma)$ in $\Gamma_{t} / \gamma_{k}\left(\Gamma_{t}\right)$ equals $\Gamma_{t} / \gamma_{k}\left(\Gamma_{t}\right)$. This shows that $\varphi_{(\infty, k)}$ is surjective.

\section{Universality of $\Gamma_{\infty}$}

In this section we prove Theorem 5 of the introduction. 
Proof To facilitate the discussion, and in view of the theorem we are now proving, we refer to the factorization $\Gamma \rightarrow \Gamma_{\infty, \varphi} \rightarrow G$ as the subnormal closure of $\varphi$. When the maps are understood, we refer to the group $\Gamma_{\infty, \varphi}$ itself as the subnormal closure of $\Gamma$ with respect to $G$. Notice that the subnormal closure is a functor on maps of groups and thus acts on squares as in Eq. (2.1) of Lemma 2.3, and respects compositions. We use the following

Notation 3.1 Given a commutative diagram of group homomorphisms:

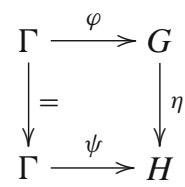

we denote by $\bar{\eta}_{\infty}$ the induced map on the subnormal closures:

$$
\bar{\eta}_{\infty}: \Gamma_{\infty, \varphi} \rightarrow \Gamma_{\infty, \psi}
$$

Throughout this proof $\Gamma_{\infty}$ denotes $\Gamma_{\infty, \varphi}$. We begin the proof by noting that by Theorem 2.11, $\Gamma_{\infty}$ is finite. Further, by Theorem 2.11 , the map $\rho: \Gamma \rightarrow \mathcal{C}$, where $\mathcal{C}$ is as in Notation 2.2(1), satisfies $\Gamma_{\infty}=\Gamma_{t, \rho}=\Gamma_{\infty, \rho}$, for some integer $t \geq 1$. By the same theorem we have a finite tower of normal maps, the first $r$ being normal inclusions leading from the subnormal closure $\mathcal{C}$ to $G$ :

$$
\Gamma_{\infty}=\Gamma_{t, \rho} \stackrel{\rho_{t-1}^{-}}{\longrightarrow} \Gamma_{t-1, \rho} \cdots \rightarrow \Gamma_{2, \rho} \rightarrow \mathcal{C}=C_{r} \hookrightarrow C_{r-1} \hookrightarrow \cdots \hookrightarrow G
$$

This implies that the canonical map:

$$
l: \Gamma_{\infty} \rightarrow G
$$

is a subnormal map, by definition. But by lemma 4.2 of [6] the crossed module closure of the image of the map $\varphi_{\infty}: \Gamma \rightarrow \Gamma_{\infty}=\Gamma_{t, \rho}$, is $\Gamma_{\infty}$. Since the tower (3.1) is finite and terminates at $\Gamma_{\infty}$, we have

$$
\Gamma^{\varphi_{\infty}} \rightarrow \Gamma_{\infty}
$$

is the trivial extension and we take this map as the identity. Of course this implies that the crossed module closures tower of $\varphi_{\infty}: \Gamma \rightarrow \Gamma_{\infty}$ is constant, so

$$
\bar{l}_{\infty}: \Gamma_{\infty, \varphi_{\infty}} \longrightarrow \Gamma_{\infty}
$$

is the identity map.

Now we show that the map $l$ is initial among all subnormal factorization maps of $\varphi$. Let $\Gamma \stackrel{\psi}{\rightarrow} S \stackrel{s}{\rightarrow} G$ be a factorization of our map $\varphi$ via a subnormal map $s: S \rightarrow G$. 
We need to show that there is a unique map $\tilde{s}: \Gamma_{\infty} \rightarrow S$ rendering the following diagram commutative.

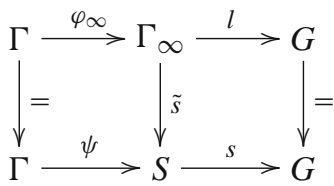

To see this we consider the map induced on the subnormal closures by the given subnormal map $s$ :

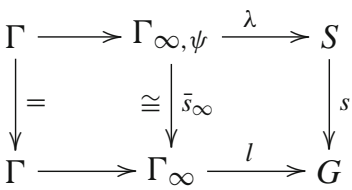

Here $\bar{s}_{\infty}$ is the map induced by naturality of the subnormal closure as in Lemma 2.3, and $\Gamma_{\infty, \psi}$ is the subnormal closure of the map $\psi: \Gamma \rightarrow S$. Since $\bar{s}_{\infty}$ is an isomorphism by Proposition 2.4, one gets a well defined map

$$
\tilde{s}=\lambda \circ\left(\bar{s}_{\infty}\right)^{-1}: \Gamma_{\infty} \rightarrow S .
$$

To see that this latter map is unique, consider any map $\sigma: \Gamma_{\infty} \rightarrow S$ as in diagram (3.2), with $\tilde{s}$ replaced with $\sigma$. This map $\sigma$ induces, by naturality, the following commutative diagram of groups, where the two lower squares do not depend on the choice of $\sigma$ :

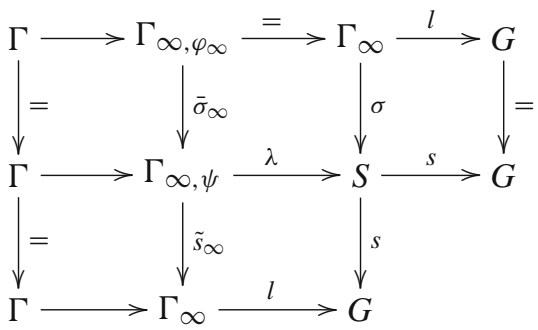

Now we rewrite the map $\sigma$ in terms of the maps in the given decomposition $\Gamma \rightarrow$ $S \rightarrow G$ alone: we read in the middle upper square: $\sigma=\bar{\sigma}_{\infty} \circ \lambda$. But since:

$$
\bar{\sigma}_{\infty} \circ \bar{s}_{\infty}=\overline{(\sigma \circ s)}_{\infty}=\bar{l}_{\infty}=i d
$$

It follows that both $\bar{\sigma}_{\infty}$, being the inverse to $\bar{s}_{\infty}$, and thus $\sigma$ itself, are determined by $\lambda$-constructed out of $\psi$ and $s$ as claimed.

\section{Examples}

In this section we give two examples. In both examples we take $G$ to be perfect with $G=\left\langle\varphi(\Gamma)^{G}\right\rangle$. In the first Example 4.1 we assume that $\Gamma$ and $G$ are finite and that 
$\operatorname{ker} \varphi \leq Z_{\infty}(\Gamma)$, and we show that $\operatorname{ker} \varphi_{\infty}$ can have arbitrarily large nilpotency class. In the second Example 4.4 we show that if $\Gamma$ is perfect, then $\Gamma_{\infty}=\Gamma_{2}$ is the universal $\varphi$-central extension of $G$ (see [7]).

Example 4.1 In this example we assume that $\Gamma$ and $G$ are finite and that $\operatorname{ker} \varphi \leq$ $Z_{\infty}(\Gamma)$. The purpose of this example is to show that the nilpotency class of $\operatorname{ker} \varphi_{\infty}$ can be arbitrarily large. We first need the following easy lemma and its corollary.

Lemma 4.2 Let $G$ be perfect and set $H:=\Gamma_{\infty}$. Then $H$ is a central product $H=H^{(\infty)} \circ Z_{\infty}(H)$. Here, $H^{(\infty)}$ is the last term of the derived series of $H$. If the nilpotent residual $\Gamma / \gamma_{\infty}(\Gamma)$ of $\Gamma$ has nilpotency class $c$, then the nilpotency class of $Z_{\infty}(H)$ is $\leq c+1$.

Proof First, by [6, Theorem 4.1], $H=\Gamma_{\infty}$ is finite, because $G=\left\langle\varphi(\Gamma)^{G}\right\rangle$. Set $L=H^{(\infty)}$ and $\mathcal{Z}=Z_{\infty}(H)$. There is a surjection $H \rightarrow G$ whose kernel is contained in $\mathcal{Z}$, and since $G$ is perfect, we get that $H=L \mathcal{Z}$. Since $L$ is perfect, $Z_{\infty}(L)=Z(L)$, and of course $[L, \mathcal{Z}] \leq Z_{\infty}(L)=Z(L)$. By the three subgroup lemma we get $[L, \mathcal{Z}]=1$, and so $H=L \circ \mathcal{Z}$.

Next, by Proposition 2.14, the nilpotent residuals of $\Gamma$ and $H$ are isomorphic. Clearly $\gamma_{\infty}(H)=L$ and $\Gamma / \gamma_{\infty}(\Gamma) \cong H / \gamma_{\infty}(H)=H / L \cong \mathcal{Z} /(\mathcal{Z} \cap L)$ is nilpotent of class $c$. Hence, since $\mathcal{Z} \cap L \leq Z(\mathcal{Z})$, we see that the nilpotency class of $\mathcal{Z}$ is $\leq c+1$.

Corollary 4.3 Let $G$ be perfect, and let c (resp. d) be the nilpotency class of the nilpotent residual of $\Gamma($ resp. of $K:=\operatorname{ker} \varphi)$. Then $\operatorname{ker} \varphi_{\infty}$ has nilpotency class $\geq d-c-1$.

Proof Let $f: \Gamma_{\infty} \rightarrow G$. Then ker $f \leq Z_{\infty}\left(\Gamma_{\infty}\right)$. Since $\varphi_{\infty} \circ f=\varphi$, we see that $\varphi_{\infty}(K) \leq \operatorname{ker} f \leq Z_{\infty}\left(\Gamma_{\infty}\right)$. Now $\operatorname{ker} \varphi_{\infty} \leq K$. By Lemma 4.2, the nilpotency class of $\varphi_{\infty}(K) \leq c+1$. Since $K / \operatorname{ker} \varphi_{\infty} \cong \varphi_{\infty}(K)$, and the nilpotency class of $K$ is $d$, the corollary follows.

We now construct examples where $G$ is perfect (in fact simple), $\gamma_{\infty}(\Gamma)=[\Gamma, \Gamma]$, so one has:

- The nilpotent residual of $\Gamma$ has nilpotency class $c=1$.

- The nilpotency class $d$ of $\operatorname{ker} \varphi$ is arbitrarily large.

By Corollary 4.3, the nilpotency class of $\operatorname{ker} \varphi_{\infty}$ is $\geq d-2$, so it is arbitrarily large.

Let $p$ be a prime, let $n \geq 2$ such that $p$ divides $n$ and let $q$ be a prime power such that $p$ divides $q-1$. Let $H$ be an image of $\operatorname{SL}_{n}(q)$ such that $Z(H) \cong \mathbb{Z}_{p}$ is cyclic of order $p$. Let $B$ be an elementary abelian group of order $p^{n}$, and let $\Gamma$ be the wreath product $\Gamma=H$ wr B. By [8, Sect. 3, p. 282-283], $Z_{\infty}(\Gamma)$ is of nilpotency class $(n-1) p+1$ ([8, Lemma 3.2, p. 283]). Also, $\gamma_{\infty}(\Gamma)=[\Gamma, \Gamma]$ is isomorphic to a direct product of $p^{n}$ copies of $H$ ([8, Lemma 3.1, p. 283]). Clearly $\Gamma / Z_{\infty}(\Gamma)$ is isomorphic to $\operatorname{PSL}_{n}(q) w r B$. But $\operatorname{PSL}_{n}(q) w r B$ is contained in $G=\operatorname{PSL}_{n p^{n}}(q)$. It follows that there exists a homomorphism $\varphi: \Gamma \rightarrow G$ whose kernel is $Z_{\infty}(\Gamma)$. Hence $G, \Gamma$ and $\operatorname{ker} \varphi$ have the claimed properties. 
Example 4.4 Suppose $\Gamma$ is perfect. Then under the above assumptions, $G$ is perfect and $\Gamma_{\infty}=\Gamma_{2}$ is a perfect group which is the universal $\varphi$-central extension of $G$.

Indeed, since $G=\left\langle\varphi(\Gamma)^{G}\right\rangle$, and $\varphi(\Gamma)$ is perfect (because $\Gamma$ is), $G$ is perfect. Also, by [6, Lemma 3.3], $\Gamma_{2}$ is a central extension of $G$, and $\Gamma_{2}$ is generated by $\left\{\varphi(\Gamma)^{g} \mid g \in G\right\}$, so $\Gamma_{2}$ is perfect. The same argument shows that $\Gamma_{3}$ is a perfect central extension of $\Gamma_{2}$. By [5, Prop. 1.8, p. 637], $\Gamma_{3}$ is a central extension of $G$. It is easy to check now that $\Gamma_{2}=\Gamma_{3}$, by the universal property of $\Gamma_{2}$, and that the assertion above holds.

\section{References}

1. Homotopy limits, Completions and Localizations, Lecture Notes in Math., vol 304, Springer (1972)

2. Bousfiled, A., Kan, D.: Homotopy Limits, Completions and Localizations. Springer, Berlin (1972)

3. Brown, R., Higgins, P.J.: On the connection between the second relative homotopy groups of some related spaces. Proc. London Math. Soc. 36, 193-212 (1978)

4. Brown, R., Higgins, P.J., Sivera, R.: Nonabelian Algebraic Topology, EMS Tracts in Math., vol. 15, European Mathematical Society (EMS), Zürich (2011)

5. Chachólski, W., Damian, E., Farjoun, E.D., Segev, Y.: The A-core and A-cover of a group. J. Algebra 321(2), 631-666 (2009)

6. Farjoun, E.D., Segev, Y.: Normal closure and injective normalizer of a group homomorphism. J. Algebra. http://arxiv.org/abs/1403.3501

7. Farjoun, E.D., Segev, Y.: Relative Schur multiplier and the universal extensions of group homomorphisms, submitted (2014). http://arxiv.org/pdf/1410.6090v1

8. Meldrum, J.D.P.: On central series of a group. J. Algebra 6, 281-284 (1967)

9. Robinson, D.J.S.: A Course in the Theory of Groups, 2nd addition, Grad. Texts in Math., Springer, Berlin (1996) 